\title{
Impact de la variabilité climatique et de l'anthropisation sur les écoulements de la Bénoué (nord Cameroun)
}

\author{
Valentin Brice Ebodé ${ }^{1}$, Gil Mahé ${ }^{2}$, and Ernest Amoussou ${ }^{3}$ \\ ${ }^{1}$ Département de Géographie, Université de Yaoundé 1, Cameroun \\ ${ }^{2}$ Institut de la Recherche pour le Développement, France \\ ${ }^{3}$ Département de Géographie et Aménagement du Territoire, Université de Parakou, Bénin
}

Correspondence: Valentin Brice Ebodé (ebodebriso@gmail.com)

Published: 16 November 2021

\begin{abstract}
Résumé. La sécheresse observée en Afrique tropicale vers la fin des années 1960, a également affecté le bassin de la Bénoué en Afrique centrale, avec une persistance remarquable qui s'est répercutée sur les écoulements. Les ruptures à la baisse ont été mises en évidence dans les séries hydropluviométriques de ce bassin au pas de temps annuel en 1970-1971 (pluies) et 1971-1972 (débits). Les déficits associés à cette rupture sont de -2, 9\% pour les pluies et $-14,2 \%$ pour les débits, par rapport à la moyenne climatologique (1950-1951 à 2014-2015). La saison humide a connu des évolutions pratiquement identiques. Cependant, depuis la décennie 1990, il est observé un relèvement significatif dans les écoulements moyens annuels de ce cours d'eau, et cela coïncide avec le retour des pluies. Le maintien de cette hausse au cours des récentes décennies pourrait être envisagé en raison de l'accroissement des espaces imperméabilisés dans le bassin, qui compenseraient le déficit engendré par la rechute des pluies après la décennie 1990 via une accentuation du ruissèlement. De plus, depuis la mise en eau du barrage de Lagdo en 1983, il est observé non seulement une augmentation de l'ensemble des gammes de débits minima suivant des taux allant de $+57,8 \%$ (minimum sur 1 jour) à $+70,1 \%$ (minimum sur 90 jours), mais aussi un accroissement général de la variabilité de l'ensemble des débits extrêmes (minima et maxima). L'augmentation progressive du stockage en saison de pluies à la retenue de Lagdo, pour fournir l'électricité et l'eau d'irrigation durant la saison sèche à une population grandissante, rendent imperceptibles les effets de l'accroissement des espaces imperméabilisés non seulement sur les écoulements de la saison humide, mais aussi sur les écoulements maxima. Au demeurant, l'opérationnalisation du barrage de Lagdo a eu un impact significatif sur le régime de la Bénoué, se traduisant essentiellement par le caractère désormais permanent de l'écoulement tout au long de l'année hydrologique et un important fléchissement de la crue d'août à octobre.
\end{abstract}

Abstract. A tendency to drought conditions appeared in tropical Africa from the end of 1960s, also affect the Benoue watershed in central Africa, with a remarkable persistence in streamflow. Negative breakpoints are found in the annual hydroclimatic time series of the Benoue catchment at annual time step in 1970-1971 for rainfall, and in 1971-1972 for discharges. Compared to the climatological mean (1950-1951 to 2014-2015), the deficits associated with these negative breakpoints are approximating $-2.9 \%$ for rainfall, and $-14.2 \%$ for discharges. The wet season shows similar developments. However, from the 1990s, there has been a significant increase in the mean annual discharges of the Benoue River, which coincides with that of the rainfall during the same decade. The maintenance of this increase over the recent decades could also be expected in response to an increase in impervious surface areas (ISA) in the catchment area, which could compensate the deficit generated by the post-1990s rainfall deficit through increasing in runoff. From the watering of the Lagdo dam in 1983, an increase in all ranges of minimum flow, ranging from $+57.8 \%$ ( $1 \mathrm{~d}$ minimum) to $+70.1 \%$ ( $90 \mathrm{~d}$ minimum), as well as an increase in the variability of extreme flows (minima and maxima), has been detected. During the wet season, the increase in water storage capacity at the Lagdo reservoir, which is used to provide electricity and irrigation water to a growing population, mitigates the increase in runoff, especially in maximum 
runoff. Moreover, the operationalization of the Lagdo dam has a significant impact on the Benoue hydrological regime, resulting mainly in a new perennial nature of the flow, and a significant decrease in flood from August to October.

\section{Introduction}

L'effet du déficit pluviométrique sur les ressources en eau de surface a été mis en évidence par de nombreuses études en milieu tropical africain (Paturel et al., 2003; Liénou et al., 2008; Sidibé et al., 2018, 2020; Tramblay et al., 2020; Ebodé, 2020; Ebodé et al., 2020a). Les changements climatiques observés au cours des cinquante dernières années dans cette région, notamment au nord Cameroun, induisent des impacts visibles dans le cycle hydrologique du bassin versant de la Bénoué (Fita, 2020). Outre cette variabilité climatique, qui détermine la dynamique hydrologique de cet hydrosystème, le récent phénomène d'anthropisation (aménagement hydraulique et changements d'occupation du sol) a certainement provoqué un changement des relations pluie/débit et du régime hydrologique, qui nécessite une nouvelle étude de la variabilité hydrologique. Les aménagements hydrauliques ont en effet connu un essor très important durant la décennie 1980. Cependant, nous avons à présent suffisamment de recul pour évaluer leurs performances réelles, ainsi que leurs impacts socio-environnementaux. Par exemple, la construction du barrage de Lagdo entre 1977 et 1982, et sa mise en service depuis 1983, pourrait avoir entraîné une modification du régime hydrologique de la Bénoué. Il en est de même de l'accroissement de la population observé dans le bassin (BUCREP, 2011), qui a pour corollaire l'accroissement des surfaces imperméabilisées dont le rôle sur les écoulements a largement été prouvé ailleurs (Aulenbach et al., 2017; Diem et al., 2017; Ebodé et al., 2020a).

Cette étude est consacrée à l'analyse des relations pluie/débit sur la période récente (1950-1951 à 2014-2015) dans le bassin de la Bénoué, au moyen des séries hydroclimatiques actualisées et des classifications obtenues à partir du traitement des images satellitaires. Cette étude apparaît ainsi fondamentale pour la communauté, en raison du rôle majeur de ce bassin sur la production hydroélectrique, et des disfonctionnements observés lors des dernières décennies, et souvent. En outre, les données et les nouveaux résultats de cette étude aideraient à la planification à long terme de la demande et de l'utilisation de l'eau, ainsi qu'à l'amélioration des simulations futures du débit de la Bénoué. L'objectif principal de cet article est donc de documenter le type et l'ampleur des changements de régime hydrologique de la Bénoué dus aux changements climatiques et à l'anthropisation.

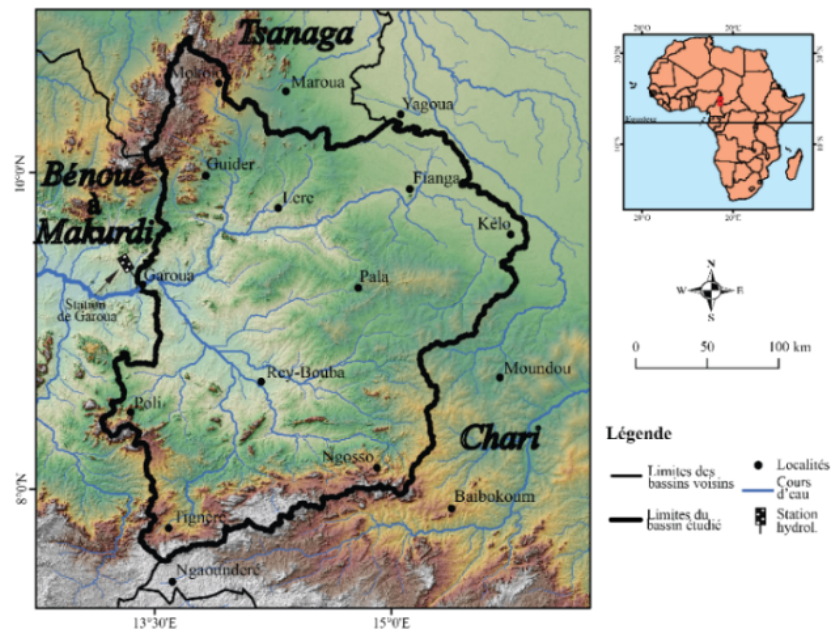

Figure 1. Localisation du bassin versant de la Bénoué.

\section{Cadre, données et méthodes}

Couvrant une aire de $61810 \mathrm{~km}^{2}$ à Garoua, le bassin de la Bénoué est situé en zone tropicale nord entre le Cameroun et le Tchad $\left(7^{\circ} 32^{\prime}-10^{\circ} 53^{\prime} \mathrm{N}\right.$ et $\left.13^{\circ} 9^{\prime}-15^{\circ} 52^{\prime} \mathrm{E}\right)$, la plus grande partie se situant sur le territoire camerounais (Fig. 1). Il est soumis à un climat tropical soudanien enregistrant en moyenne $830 \mathrm{~mm}$ de précipitations par an. Ce climat est caractérisé par une saison sèche et chaude s'étendant en moyenne de novembre à avril, et une saison humide et moins chaude de mai à octobre, au cours de laquelle se produit l'essentiel des précipitations. La saison humide enregistre à peu près $887 \mathrm{~mm} \mathrm{an}^{-1}$ de précipitations, tandis que la saison sèche n'enregistre qu'environ $43 \mathrm{~mm} \mathrm{an}^{-1}$.

Plusieurs jeux de données (hydropluviométriques et d'occupation du sol) ont été utilisés dans ce travail. Disponible sur la période 1950-2015, la série des débits de la Bénoué provient de l'Autorité du Bassin du Niger (ABN). Elle comporte d'importantes lacunes entre 1998 et 2009.

Les données de pluies utilisées dans cette étude sont celles du CRU (Climatic Research Unit) de l'université d'East Anglia au Royaume Uni. Ces données sont disponibles depuis 1901 via le site https://climexp.knmi.nl/selectfield_obs2. cgilunskiplpenalty\@M Mhskip.5\fontdimen2\font|relax?id= _2833fad3fef1bedc6761d5cba64775fo/ (la date du dernier accès : 10 mai 2021) au format NetCDF, sur un pas temps mensuel et à une résolution spatiale de $0,25^{\circ} \times 0,25^{\circ}$. 
Les données d'occupation du sol dans le bassin de la Bénoué sont issues des images satellitaires Landsat 8 de janvier à mars 2015 et Landsat TM de janvier à mars 1987. L'ensemble de ces images sont mises à disposition du grand public gratuitement par la National Aeronautics and Space Administration (NASA), via le site de l'US Geological Survey (https://earthexplorer.usgs.gov/, la date du dernier accès : 13 janvier 2020), au format GeoTIFF.

L'analyse des séries de pluies, des débits moyens et des coefficients d'écoulement a été réalisée à l'aide des tests statistiques de détection de rupture (corrélation sur le rang et test de Pettitt; Lubès et al., 1994). Dans l'optique d'apprécier le comportement des débits extrêmes, l'outil Indicators of Hydrologic Alteration (IHA), version 7.1, développé par The Nature Conservancy a été utilisé. La classification supervisée par maximum de vraisemblance des images satellitaires Landsat téléchargées, et ce, à l'aide du logiciel SNAP (https://www.theia-land.fr, la date du dernier accès : 20 février 2020), nous a permis d'effectuer une analyse diachronique de l'évolution de l'occupation du sol dans le bassin étudié.

\section{Résultats et discussion}

\subsection{Evolution des pluies annuelles et saisonnières}

Les pluies annuelles diminuent de manière statistiquement significative sur le bassin de la Bénoué. Le test de Pettitt situe dans leur série une rupture majeure intervenue en 1970-1971 (Fig. 2). Cette dernière marque le passage d'une période excédentaire, dont le surplus par rapport à la moyenne climatologique est de $+6,1 \%$, à une période déficitaire, ayant un déficit par rapport à cette même moyenne de -2, $9 \%$ (Fig. 2). L'analyse des écarts décennaux révèle une chute depuis la décennie 1970, qui n'a été périodiquement interrompue qu'au cours de la décennie 1990 (Tableau 1). Les volumes précipités durant la saison humide suivent sensiblement les mêmes tendances (Fig. 2). Notamment, ces pluies présentent également une rupture à la baisse en 1970-1971, selon le test de Pettitt. L'excédent et le déficit enregistrés de part et d'autre de cette rupture sont, respectivement, de $+5,2 \%$ et $-2,3 \%$ (Fig. 2). La décennie 1990 apparaît aussi plus excédentaire dans les pluies de cette saison par rapport aux décennies 1970 et 1980 (Tableau 1). Comme en saison humide, les volumes précipités en saison sèche diminuent significativement, selon le test de Pettitt. Une rupture a été identifiée par le test de Pettitt dans la série des pluies de la saison sèche en 1970-1971 (Fig. 2). Les excédents et déficits par rapport à la moyenne climatologique de chacune des périodes situées avant et après ladite rupture sont, respectivement, de $+21,3 \%$ et $-11,5 \%$. La chute des pluies de la saison sèche observée au cours de la décennie 1970 est continue jusqu'à la décennie 2010 (Tableau 1). Un retour à des conditions plus humides au cours de la décennie 1990 a été suggéré en Afrique de l'ouest (Alhassane et al., 2013; Descroix et al., 2020). Cela concorde avec les résultats de notre étude, qui mettent en évidence un rehaussement des précipitations annuelles au cours de la décennie 1990, consécutif à la sécheresse des décennies 1970 et 1980. Cependant le rehaussement signalé dans cette étude concerne uniquement cette décennie, tandis que celui relevé en Afrique de l'ouest persiste jusqu'à présent (Descroix et al., 2020).

\subsection{Dynamique de l'occupation du sol dans le bassin de la Bénoué}

Les classifications effectuées à partir des images Landsat des années 1987 et 2015 montrent d'importants changements dans les principaux modes d'occupation du sol ayant un lien direct avec les écoulements dans le bassin versant de le Bénoué (Fig. 3). En 28 ans, il a été enregistré une diminution des surfaces forestières (îlots de forêt sous forme de petits massifs ou de forêts galeries et savanes boisées) de $24 \%$ (Tableau 2). Inversement, les espaces imperméabilisés (bâti, voirie, sols nus et zones cultivées) et les plans d'eau (lacs, étangs et grandes rivières) ont augmenté, respectivement, de $133 \%$ et de $16,4 \%$ (Tableau 2).

\subsection{Les répercussions des forçages environnementaux sur les écoulements}

\subsubsection{Le rôle de la pluviométrie}

Les débits moyens annuels de la Bénoué ont enregistré une rupture à la baisse en 1971-1972. Le déficit par rapport à la moyenne de la période étudiée observé suite à cette rupture est de $-14,2 \%$ (Fig. 2). L'impact de la pluviométrie dans l'évolution des écoulements de la Bénoué semble assez net à ce pas de temps. On note pour les deux variables comparées des diminutions statistiquement significatives sur la période d'étude (Fig. 2). A ce pas de temps, la stationnarité du régime hydrologique, et celui des précipitations, obéit à la logique générale qui admet que les ruptures hydrologiques interviennent à la suite des ruptures pluviométriques, puisqu'une rupture à la baisse est intervenue dans la série des précipitations annuelles en 1970-1971 et dans celle des débits moyens annuels en 1971-1972 (Fig. 2). Comme au pas de temps annuel, l'influence de la pluviométrie de la saison humide est également perceptible dans les écoulements de la même saison (Fig. 2). On note alors, dans les séries respectives de pluies et débits, des ruptures à la baisse, avec une légère précocité pour les pluies (1970-1971), permettant d'envisager un éventuel rôle de la diminution des pluies sur celle des écoulements.

\subsubsection{L'impact du barrage}

Sur le bassin de la Bénoué, depuis la mise en eau du barrage de Lagdo (1983), on note une augmentation importante de l'ensemble des gammes de débits minima sur des jours consécutifs (Tableau 3). Ces augmentations sont comprises 

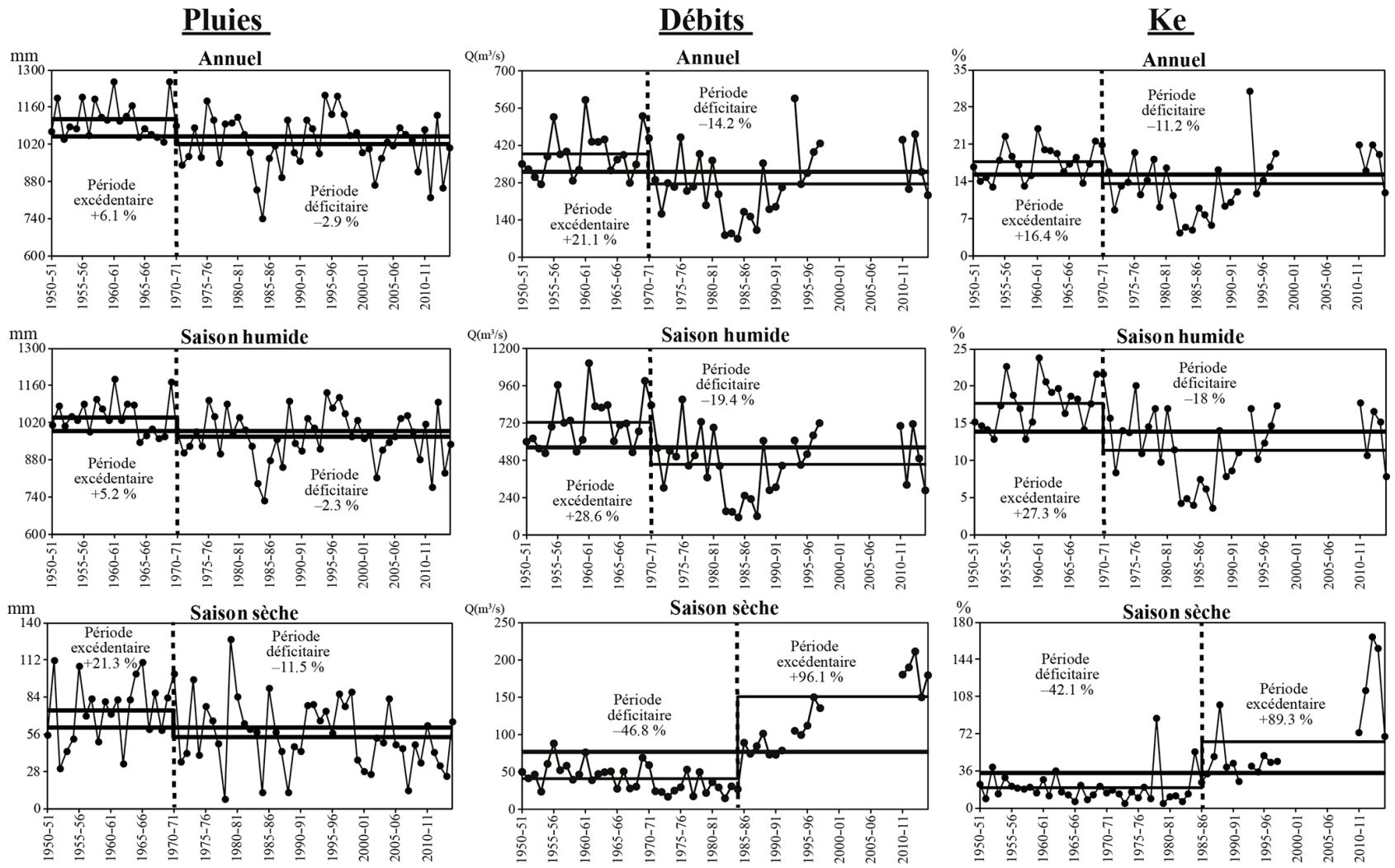

Figure 2. Evolution des pluies, débits et coefficients d'écoulement (Ke) annuels et saisonniers de la Bénoué entre 1950-1951 et 2014-2015. Traits horizontaux fins (épais) : moyenne des périodes homogènes (moyenne climatologique), tireté vertical : année de rupture.

Tableau 1. Ecarts $(\%)$ des moyennes décennales annuelles et saisonnières des pluies, débits et coefficients d'écoulement par rapport à leurs moyennes climatologiques sur le bassin de la Bénoué.

\begin{tabular}{|c|c|c|c|c|c|c|c|c|c|}
\hline \multirow[b]{2}{*}{ Décennies } & \multicolumn{3}{|c|}{ Pluies } & \multicolumn{3}{|c|}{ Débits } & \multicolumn{3}{|c|}{$\mathrm{Ke}$} \\
\hline & Annuel & $\begin{array}{l}\text { Saison } \\
\text { humide }\end{array}$ & $\begin{array}{r}\text { Saison } \\
\text { sèche }\end{array}$ & Annuel & $\begin{array}{l}\text { Saison } \\
\text { humide }\end{array}$ & $\begin{array}{r}\text { Saison } \\
\text { sèche }\end{array}$ & Annuel & $\begin{array}{l}\text { Saison } \\
\text { humide }\end{array}$ & $\begin{array}{r}\text { Saison } \\
\text { sèche }\end{array}$ \\
\hline 1950 & 6 & 5,7 & 11,9 & 11,3 & 16,6 & $-34,2$ & 7 & 15,3 & $-39,2$ \\
\hline 1960 & 6,3 & 5,1 & 26,2 & 29,2 & 38,5 & $-39,3$ & 23 & 36,5 & $-48,5$ \\
\hline 1970 & 0 & $-0,4$ & 5,2 & $-6,5$ & 0,6 & $-58,5$ & $-4,8$ & 4,7 & $-41,5$ \\
\hline 1980 & $-7,3$ & $-6,9$ & $-13,4$ & $-43,7$ & -46 & $-27,3$ & $-40,5$ & $-42,2$ & 2,3 \\
\hline 1990 & 4 & 3,5 & 11,9 & 9,9 & $-6,4$ & 128,8 & 8 & $-6,5$ & 20,5 \\
\hline 2000 & $-5,2$ & $-3,7$ & $-29,7$ & - & - & - & - & - & - \\
\hline 2010 & $-6,4$ & $-5,1$ & $-26,6$ & 7,1 & $-10,6$ & 136,7 & 16,7 & $-2,4$ & 242,7 \\
\hline
\end{tabular}

entre $57,8 \%$ (débit minimum sur 1 jour) et $70,1 \%$ (débit minimum sur 90 jours). Au-delà du retard moyen de cinq jours noté dans l'apparition des minima (Tableau 3), il est également noté un accroissement de la variabilité interannuelle pour les deux catégories de débits extrêmes évaluées (maxima et minima), qui semble beaucoup plus important pour les maxima (Tableau 3). L'ensemble de ces évolutions semblent liées au fonctionnement du barrage. Cet ouvrage permet en effet de stocker de l'eau en saison humide et de la relâcher en saison sèche, dans l'optique de continuer la production hydroélectrique et l'irrigation en aval durant cette saison, modifiant ainsi le comportement naturel de ce cours d'eau. La hausse des volumes lâchées (également perceptible au travers des minimums) sans cesse croissante au fil du temps (Fig. 4), traduit un accroissement de plus en plus important de la population et de ses besoins grandissants en énergie auxquels il faut subvenir en permanence, y compris en saison sèche. Le barrage de Lagdo a considérablement affecté le régime de la Bénoué. Depuis sa mise en place, on note le développement d'un caractère permanent de l'écou- 


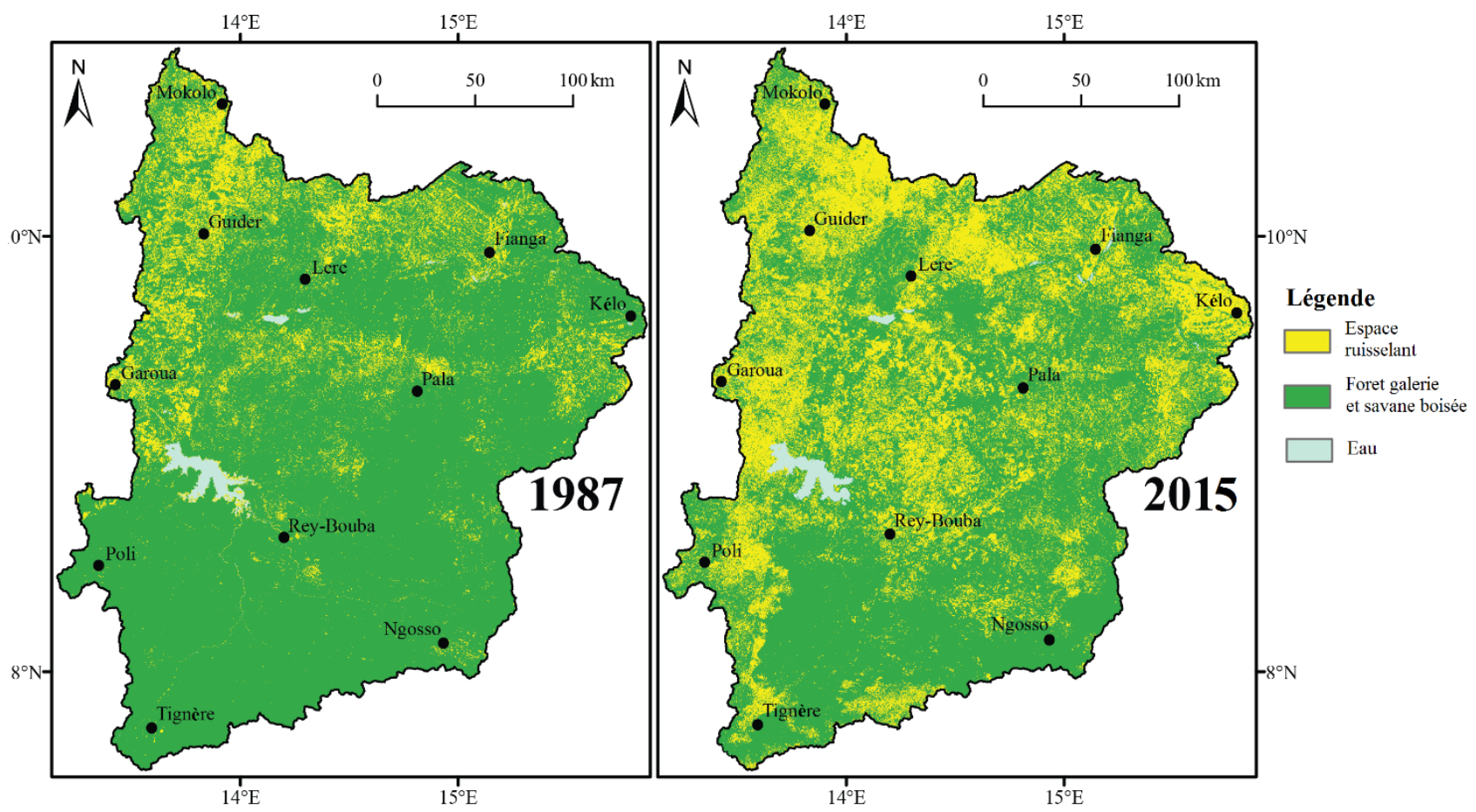

Figure 3. Principaux modes d'occupation du sol dans le bassin de la Bénoué en 1987 et 2015.

Tableau 2. Evolution des principaux modes d'occupation du sol dans le bassin de la Bénoué entre 1987 et 2015.

\begin{tabular}{lrrrrr}
\hline Modes d'occupation du sol & \multicolumn{2}{c}{ Superficie dans le bassin $\left(\mathrm{km}^{2}\right)$} & & Evolution entre 1987 et 2015 \\
\cline { 2 - 3 } \cline { 5 - 6 } & 1987 & 2015 & & $\mathrm{~km}^{2}$ & $\%$ \\
\hline Forêt galerie et savane boisée & 51977 & 59480 & & -12497 & -24 \\
Plan d'eau & 505 & 588 & & 83 & 16,4 \\
Espace ruisselant & 9328 & 21742 & & 12414 & 133 \\
\hline
\end{tabular}

lement tout au long de l'année hydrologique, ainsi que le fléchissement de la crue d' août à octobre (Fig. 4). La hausse des débits d'étiage est identifiée comme conséquence majeure des aménagements hydrauliques (barrages), comme proposé précédemment par Amoussou et al. (2012).

\subsubsection{La contribution des changements des modes d'occupation du sol}

Il est certes vrai que les écoulements de la Bénoué diminuent statistiquement depuis le début de la décennie 1970, en rapport avec la chute des précipitations entamée durant cette même décennie. Cependant, l'analyse des écarts décennaux confirme un relèvement important des écoulements depuis la décennie 1990. Les écarts relevés sont de $+9,9 \%$ pour la décennie 1990, et $+7,1 \%$ pour la décennie 2010 (Tableau 1). Il est tout à fait possible d'associer la hausse des écoulements de la décennie 1990 à celle de la pluviométrie. Par contre, rien n'explique le maintien de cette hausse sur les récentes décennies, puisque les pluies diminuent durant cette période. Les déficits calculés sont de $-5,2 \%$ pour la décennie 2000 et $-6,4 \%$ pour la décennie 2010 (Tableau 1). Nous pouvons suggérer que la hausse des écoulements, associée à l'augmentation des pluies dans la décennie 1990, a été immédiatement relayée par l'accroissement des espaces imperméabilisés dans le bassin. Ces formations imperméables agissent dans le sens d'un accroissement du ruissellement et, par conséquent, des écoulements. Des études effectuées ailleurs sont parvenus à des résultats similaires (Aulenbach et al., 2017 ; Ebodé et al., 2020a). Il est aussi tout à fait possible de s'interroger sur les causes de l'augmentation récente des écoulements observée sur le bassin de la Bénoué, et en particulier sur son absence durant la saison humide et dans les maxima. L'augmentation de plus en plus importante des quantités d'eau stockées en saison de pluies, et de celle relâchées en saison sèche (Fig. 4), pourraient être en rapport avec cette situation. Cette augmentation du stockage en saison de pluies à Lagdo implique que le surplus d'eau, occasionné par le ruissellement, ne soit pas perceptible dans les écoulements de la saison de pluies et les maxima à Garoua. 
Tableau 3. Statistiques des débits maxima et minima de la Bénoué avant et après le barrage.

\begin{tabular}{|c|c|c|c|c|c|c|}
\hline \multirow[t]{2}{*}{ Statistiques IHA } & \multicolumn{2}{|c|}{ Moyennes $\left(\mathrm{m}^{3} \mathrm{~s}^{-1}\right)$} & \multicolumn{2}{|c|}{$\mathrm{Cv}(\%)$} & \multicolumn{2}{|c|}{ Variation } \\
\hline & Avant & Après & Avant & Après & $\mathrm{m}^{3} \mathrm{~s}^{-1}$ & $\%$ \\
\hline \multicolumn{7}{|l|}{ Débits minimum } \\
\hline Minimum sur 1 jour & 0,6 & 58,4 & 1,13 & 0,96 & 57,8 & 9633 \\
\hline Minimum sur 3 jours & 0,7 & 59,7 & 0,9 & 0,96 & 59 & 8428 \\
\hline Minimum sur 7 jours & 0,8 & 61,6 & 0,85 & 0,95 & 60,8 & 7600 \\
\hline Minimum sur 30 jours & 1,5 & 66,6 & 0,84 & 0,92 & 65,1 & 4340 \\
\hline Minimum sur 90 jours & 6,1 & 76,2 & 0,6 & 0,89 & 70,1 & 1149 \\
\hline \multicolumn{7}{|l|}{ Débits maximum } \\
\hline Maximum sur 1 jour & 2957 & 1589 & 0,34 & 0,58 & -1368 & $-46,2$ \\
\hline Maximum sur 3 jours & 2851 & 1540 & 0,33 & 0,58 & -1311 & -46 \\
\hline Maximum sur 7 jours & 2608 & 1436 & 0,32 & 0,57 & -1172 & -45 \\
\hline Maximum sur 30 jours & 1979 & 1143 & 0,32 & 0,57 & -836 & $-42,2$ \\
\hline Maximum sur 90 jours & 1274 & 763,6 & 0,32 & 0,54 & $-510,4$ & -40 \\
\hline \multicolumn{7}{|c|}{ Dates juliennes moyennes } \\
\hline Du minimum & 113 & 118 & & & & \\
\hline Du maximum & 252 & 245 & & & & \\
\hline
\end{tabular}

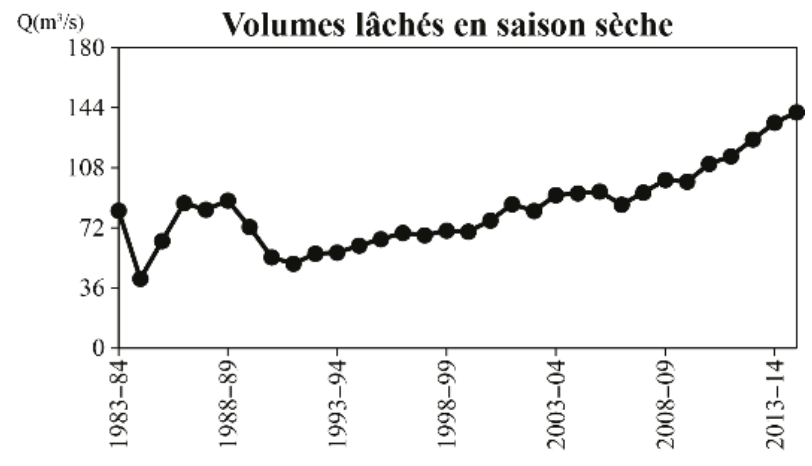

$\mathrm{Q}\left(\mathrm{m}^{7} / \mathrm{s}\right)$

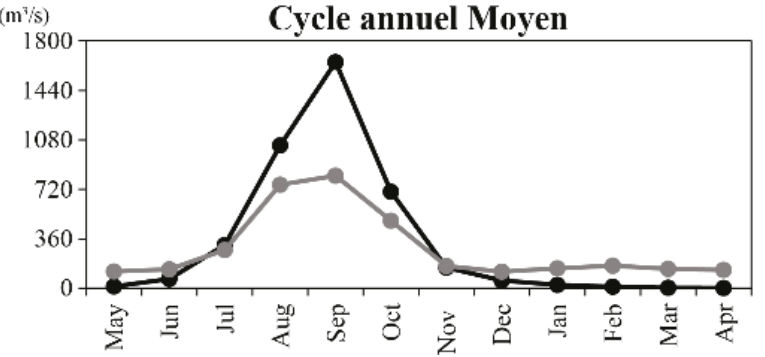

Figure 4. Evolution interannuelle des volumes lâchés à Lagdo depuis 1983 et cycle annuel moyen de la Bénoué avant (en noir) et après (en gris) le barrage.

\section{Conclusion}

A partir de la décennie 1970, le bassin de la Bénoué a connu une diminution générale des précipitations qui n'a été interrompue qu'au cours de la décennie 1990. Ce changement intervenu dans l'évolution des pluies a entraîné une mo- dification des écoulements de son collecteur principal. Ainsi, il a été relevé, depuis 1971-1972, une diminution des écoulements moyens de la saison de pluies et annuels, dont les déficits respectifs par rapport à leurs moyennes climatologiques atteignent $-19,4 \%$ et $-14,2 \%$. Certes en diminution depuis la décennie 1970, les écoulements moyens annuels augmentent depuis la décennie 1990. La hausse des écoulements de la décennie 1990 est imputable à son abondante pluviométrie, mais son maintien au cours des récentes décennies semble être la conséquence des changements de modes d'occupation du sol, puisque, durant cette période, la pluviométrie baisse. La hausse des minima observés sur ce cours d'eau, ainsi que l'accentuation de la variabilité des écoulements extrêmes semblent être associées à la mise en service du barrage de Lagdo depuis 1983. Cet ouvrage permet en effet de stocker de l'eau en saison de pluies et de la relâcher en saison sèche, dans l'optique de continuer de produire l'hydroélectricité et d'irriguer les champs situés en aval. L'augmentation dudit stockage pour le comblement des besoins d'une population grandissante pourrait d'ailleurs être à l'origine d'une diminution des crues à Garoua.

Disponibilité du code. Les logiciels utilisés dans cette étude sont gratuits. Les différents liens proposés plus haut dans cet article donnent accès aux sites sur lesquels ils peuvent être téléchargés.

Disponibilité des données. Certaines données (débits) utilisées dans cette étude proviennent d'une institution (Autorité du Bassin du Niger). Leur collecte nécessite des moyens financiers conséquents. Cette institution a la responsabilité entière sur ces don- 
nées. Pour entrer en possession de ces données, il faudrait entrer en contact avec cette institution.

D'autres données (précipitations et images satellitaires) par contre, sont disponibles gratuitement en ligne sur des sites proposés plus haut dans cet article.

Collaborateurs. Ce travail a été conceptualisé par VBE. La collecte, le traitement et l'analyse des données ont été effectués par VBE. Le projet a été dirigé par GM. La rédaction du manuscrit et la relecture ont été faites par l'ensemble des auteurs.

Intérêts concurrents. Les auteurs déclarent qu'ils n'ont aucun conflit d'intérêts.

Clause de non-responsabilité. Publisher's note : Copernicus Publications remains neutral with regard to jurisdictional claims in published maps and institutional affiliations.

Déclaration du numéro spécial. This article is part of the special issue "Hydrology of Large River Basins of Africa". It is a result of the 4th International Conference on the "Hydrology of the Great Rivers of Africa", Cotonou, Benin, 13-20 November 2021.

\section{Références}

Alhassane, A., Salack, S., Ly, M., Lona, I., Traore, S. B., et Sarr, B. : Evolution des risques agroclimatiques associés aux tendances récentes du régime pluviométrique en Afrique de l'Ouest soudano-sahélienne, Sécheresse, 24, 282293, https://doi.org/10.1684/sec.2013.0400, 2013.

Amoussou, E., Camberlin, P., et Mahé, G. : Impact de la variabilité climatique et du barrage Nangbéto sur l'hydrologie du système Mono-Couffo (Afrique de l'ouest), J. Sci. Hydrol., 57, 805-817, https://doi.org/10.1080/02626667.2011.643799, 2012.

Aulenbach, B. T., Landers, M. N., Musser, J. W., and Painter, J. A. : Effects of impervious area and BMP implementation and design on storm runoff and water quality on eight small watersheds, J. Amer. Wat. Ress. Ass., 53, 382-399, https://doi.org/10.1111/1752-1688.12501, 2017.

BUCREP : Rapport de présentation des résultats définitifs du recensement de la population en 2005, Yaoundé, Cameroun, 66 pp., 2011.

Descroix, L., Sané, Y., Mamadou, T., Manga, S.-P., Boubacar, D., Mingou, J., Mendy, V., Coly, S., Dièye, A., Badiane, A., Senghor, M.-J., Diedhiou, A.-B., Djiby, S., Bouaita, Y., Soumaré, S., Diop, A., Bakary, F., Bamol, A., Machu, E., Montoroi, J.-P., Andrieu, J., and Vandervaere, J.-P. : Inverse estuaries in West Africa : Evidence of the rainfall recovery ?, Water, 12, 647, https://doi.org/10.3390/w12030647, 2020.
Diem, J. E., Hill, T. C., and Milligan, R. A. : Diverse multi-decadal changes in streamflow within a rapidly urbanizing region, J. Hydrol., 556, 61-71, https://doi.org/10.1016/j.jhydrol.2017.10.026, 2017.

Ebodé, V. B. : Variabilité hydroclimatique en Afrique centrale occidentale forestière : entre analyse des fluctuations observées, recherche des facteurs explicatifs et modélisation prédictive, Generis Publishing, 382 p., 2020.

Ebodé, V. B., Mahé, G., Dzana, J. G., and Amougou, J. A. : Anthropization and climate change : impact on the discharges of forest watersheds in Central Africa, Water, 12, 2718, https://doi.org/10.3390/w12102718, 2020a.

Ebodé, V. B., Mahé, G., Dzana, J. G., Amougou, J. A., et Batha, R. A. : Changement climatique dans le bassin versant de l'Ogooué : évolution récente et impact sur les écoulements, Afr. Sci., 17, 110-125, 2020b.

Fita, D. E. : Impact de la variabilité climatique sur la réponse hydrologique du bassin versant supérieur de la Bénoué (nordCameroun), Thèse de doctorat, Université de Maroua, Cameroun, 2020.

Liénou, G., Mahé, G., Paturel, J. E., Servat, E., Sighomnou, D., Ekodeck, G. E., Dezetter, A., et Dieulin, C. : Evolution des régimes hydrologiques en région équatoriale camerounaise : un impact de la variabilité climatique en zone équatoriale ?, J. Sci. Hydrol., 53, 789-800, https://doi.org/10.1623/hysj.53.4.789, 2008.

Lubès, H., Masson, J. M., Servat, E., Paturel, J. E., Kouame, B., et Boyer, J. F. : Caractérisation des fluctuations dans une série chronologique par applications de tests statistiques, Etudes bibliographiques; Programme ICCARE, Rapport no. 3, ICCARE, ORSTOM, Montpellier, France, 1994.

Paturel, J. E., Ouédraogo, M., Mahé, G., Servat, E., Dezetter, A., and Ardoin, S. : Influence of the spatialization of data on the modelling of monthly river regimes in West Africa, Hydrol. Sci. J., 48, 881-890, https://doi.org/10.1623/hysj.48.6.881.51422, 2003.

Sidibé, M., Dieppois, B., Mahé, G., Paturel, J. E., Amoussou, E., Anifowose, B., and Lawler, D. : Trend and variability in a new, reconstructed streamflow dataset for West and Central Africa, and climatic interactions, 1950-2005, J. Hydrol., 561, 578-493, https://doi.org/10.1016/j.jhydrol.2018.04.024, 2018.

Sidibé, M., Dieppois, B., Eden, J., Mahé, G., Paturel, J. E., Amoussou, E., Van de Wiel, M., Anifowose, B., and Lawler, D. : Nearterm impacts of climate variability and change on hydrological systems in West and Central Africa, Clim. Dynam., 54, 20412070, https://doi.org/10.1007/s00382-019-05102-7, 2020.

Tramblay, Y., Villarini, G., and Zhang, W. : Observed changes in flood hazard in Africa, Environ. Res. Lett., 15, 1040b5, https://doi.org/10.1088/1748-9326/abb90b, 2020. 